\title{
Phacoemulsification with Peripheral Iridectomy and Trabeculectomy: Comparing the Two Procedures in Management of Angle-Closure Glaucoma
}

\author{
Asaad Nooreldin
}

Ophthalmology Department, Faculty of Medicine, Al-Azhar University (Assuit), Egypt

Corresponding author: Asaad Nooreldin, E-mail:asaad_nooreldin@yahoo.com, Mob: +201026366024

\begin{abstract}
Background: glaucoma was ranked as one of the major causes of blindness worldwide. Angle-closure glaucoma (ACG) is a type of glaucoma characterized by narrowing or closure of the anterior chamber angle.

Objective: to compare the clinical outcomes of phacoemulsification (Phaco) with peripheral iridectomy versus trabeculectomy in management of ACG. Patients and Methods: this study was a prospective, follow-up nonrandomized clinical trial, carried out on 50 eyes with ACG. Patients were divided into two groups: group A $(n=25)$; eyes underwent phacoemulsification with peripheral iridectomy, and group $\mathrm{B}(n=25)$; eyes underwent sub-scleral trabeculectomy. Intraocular pressure (IOP), visual outcomes and postoperative complications were assessed. Patients were followed-up for 6 months. Results: postoperative visual acuity was significantly improved in phaco group compared to that of trabeculectomy group $(\mathrm{P}<0.001)$. The mean postoperative IOP was reduced significantly $(\mathrm{P}<0.05)$ in both groups at all time intervals compared to their preoperative values. Anterior chamber depth increased significantly postoperatively in group (A) $(\mathrm{p}<0.001)$ however, almost no change was noticed in group (B). Meanwhile, postoperative angle opening distance was increased $(\mathrm{p}<0.001)$ in both groups. No significant differences were found between both procedures in Rim area, Rim volume, cup/disc area ratio and maximum cup depth. Eight cases (32.0\%) in group (A) and 7 cases (28.0\%) in group (B) suffered from postoperative complications with insignificant difference. Conclusion: both procedures are effective in lowering IOP in patients with ACG, but phacoemulsification with peripheral iridectomy significantly improved visual acuity compared to sub-scleral trabeculectomy with almost the same rate of complications.
\end{abstract}

Keywords: Phacoemulsification, Peripheral Iridectomy, Trabeculectomy, Angle Closure-Glaucoma.

\section{INTRODUCTION}

Glaucoma was ranked as the second most common cause of blindness following cataract and as the major cause of irreversible blindness, the World Health Organization estimated that about 20 million people will be affected by primary angle-closure glaucoma (PACG) by 2020, and 5.3 million will suffer from blindness ${ }^{(\mathbf{1})}$. Angle-closure glaucoma (ACG) is a type of glaucoma characterized by narrowing or closure of the anterior chamber angle. With increasing age, some factors causing increased shallowness of the anterior chamber and iridolenticular contact witch increased intraocular pressure (IOP) and could lead to damaging the optic nerve and losing visual field ${ }^{(2)}$.

Several studies reported that cataract extraction could restore the vision and eliminate a narrow angle in patients with chronic primary angle-closure glaucoma or acute primary angle closure ${ }^{(3)}$. Phacoemulsification (Phaco) has been usually described for treating PACG associated with cataract and it represents nowadays the gold standard for cataract surgery, for the objective of reducing IOP and improving visual acuity. But, some factors could make the use of Phacoemulsification in treating ACG be difficult such as large lens, narrow pupil and the narrow working space in the anterior chamber ${ }^{(4)}$. Peripheral iridotomy is a standard procedure commonly used for the treatment of angle closure, it ameliorates pupillary block "the common cause of angle closure".
There is an evidence demonstrates that peripheral iridotomy is possibly useful in the treatment of the early stages of PACG, but it is probably not effective in reducing intraocular pressure in case of development of glaucomatous optic neuropathy and extensive synechial angle closure ${ }^{(5)}$.

Trabeculectomy is a surgical procedure that has been first described as an antiglaucoma procedure by Cairns, in $1968^{(6)}$; this modalityis effective in the treatment of various types of glaucoma and is a popular procedure has undergone numerous modifications ${ }^{(7)}$. Trabeculectomy effectively reduces intraocular pressure and improving visual acuity in most patients in short term but by long term, these patients may need additional medical or surgical IOP control ${ }^{(8)}$. Although the significant success of trabeculectomy, it may cause some complications related to hypotony and may result in loss of visual acuity in patients with advanced field $\operatorname{loss}^{(9)}$. Both phacoemulsification and trabeculectomy have an effective role in reducing IOP in patients with medically uncontrolled chronic angle-closure glaucoma $^{(10)}$.

\section{OBJECTIVE OF THE STUDY}

The objective of this study is to compare the clinical outcomes of phacoemulsification with peripheral iridectomy versus trabeculectomy in treatment of angle-closure glaucoma. 


\section{PATIENTS AND METHODS}

This study is a prospective, follow-up nonrandomized clinical trial, which was conducted between January and December 2017 in department of Ophthalmology, Al-Azhar University hospital in Assiut city. The study included fifty eyes of forty-eight patients with angle closure glaucoma with or without cataract. They were divided into two groups: group A $(n=25)$ : eyes underwent phacoemulsification with peripheral iridectomy, and group B $(n=25)$ : eyes underwent sub-scleral trabeculectomy.

Inclusion criteria were as follows: primary angle closure (PAC) with IOP $\geq 30 \mathrm{mmHg}$ at diagnosis untreated or under medical treatment for at least six months, patient must be phakic in the affected eye (s), the patients should be 50 years or older, gonioscopy, revealed interrupted areas of peripheral anterior synachiae (PAS) (goniosynaechiae), and degree of angle closure <180 in (group A) and 180 -270 degree in (group B). While, exclusion criteria were; small palpebral aperture - difficult access, inflamed eye (eyes with uncontrolled raised IOP or eyes on chronic medical treatment), advanced glaucoma (cup-disc-ratio $\geq 0.9$ ), increased surgical risk (e.g., corneal opacity, Fuch's endothelial dystrophy; pseudoexfoliation, previous vitreo-retinal surgery), any intraocular procedure or laser treatment, Axial length $\leq 19 \mathrm{~mm}$ (nanophthalmos), history of retinal ischaemia, macular oedema or wet age-related macular degeneration, and medically unfit for surgery.

\section{Preoperative considerations:}

All patients were subjected to full ophthalmic examination including visual Acuity (VA) which was measured by the Landolt $\mathrm{C}$ chart, and the value was converted to the logarithm of the minimum angle of resolution ( $\log$ MAR), assessment of angle with goniolens, assessment of AC depth using contact Ascan, careful monitoring of intraocular pressure using applanation tonometry, assessment of fundus and optic disc cupping (C/D) ratio using slit lamp biomicroscopy (+78 D), optical coherence tomography (OCT) for the measurement of rim area and rim volume, angle opening distance (AOD) at $500 \mathrm{um}$ anterior to the scleral spur (AOD 500) and AOD at 750 um anterior to the scleral spur (AOD 750). In addition to careful evaluation and identification of risk factors such as; state of corneal health, asymmetric anterior chamber depth, phacodonesis, iridodonesis, evidence of lens subluxation, lens tilting, suprachoroidal hemorrhage in the fellow eye or previous occurrence of malignant glaucoma. Also, the intraocular lens (IOL) power was calculated by IOL master. After the diagnosis of primary angle closure glaucoma, all patients received first-line medical treatment for IOP control including a combination of topical beta-blockers, brimonidine, carbonic anhydrase inhibitors (CAIs), and additional systemic hyperosmotic agents (intravenous mannitol, 1 $\mathrm{mg} / \mathrm{kg}$ ) and CAIs (acetazolamide, $250 \mathrm{mg}$ four times daily).

\section{Surgical procedures:}

All procedures were done in operative room under complete aseptic conditions.

\section{Phacoemulsfication:}

Preoperative measures were taken prior to the beginning of the procedure. Topical anesthetic was instilled into conjunctival sac, local anaesthesiaperibulbar block was given through skin, povidone-iodine $5 \%$ or chlorhexidine was instilled into conjunctival sac and also used to paint the skin of the eyelids. Careful draping was performed ensuring that the lashes and lid margins are isolated from surgical fields and Speculum was inserted. The main incision was clear cornel; aside port incision was made around 60 to the left of the main incision. Continuous curvilinear Capsulorhexis was performed with cystotome (Figure 1) or capsule forceps and involve two movement, shearing in which atangential vector force was applied along the direction of the tear, ripping in which centripetal vector force strains and tear the capsule. Hydrodissection was performed to separate the nucleus and cortex from the capsule so that the nucleus could be more easily and safety rotated, 26-gauge blunt cannula with fluid was inserted just beneath the edge of the rhexis and fluid was injected gently under the capsule, hydro dissection wave should be seen provided there is good red reflex.

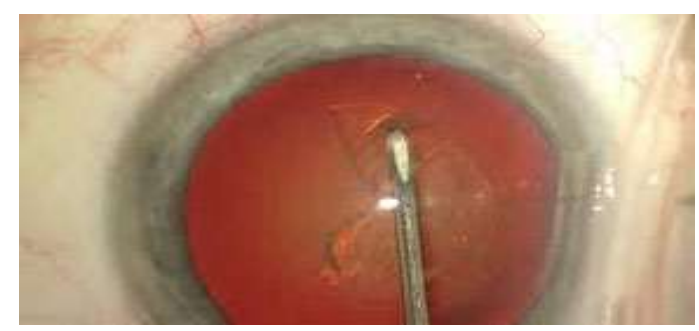

Figure (1): Capsulorrhexius with cysttome.

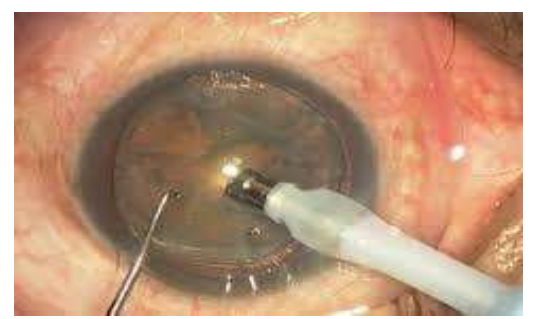

Figure (2): Nuclear management. 
Nuclear phaco chop: Vertical chopping was performed with pointed-tip chopper, the nucleus was chopped into several pieces each of which was emulsified and aspirated (Figure 2). Regarding cortical clean up, the cortical fragments were engaged by vacuum /pulled centrally and aspirated by manual aspiration method or bimanual automated method (Figure 3). Foldable hydrophilic IOL was implanted in the capsular bag followed by washing of the viscoelastic and hydration of the wound (Figure 4). Peripheral iridectomy was performed using capsulorhexisforeceps and scissors to cut a section of iris at 12 o'clock.

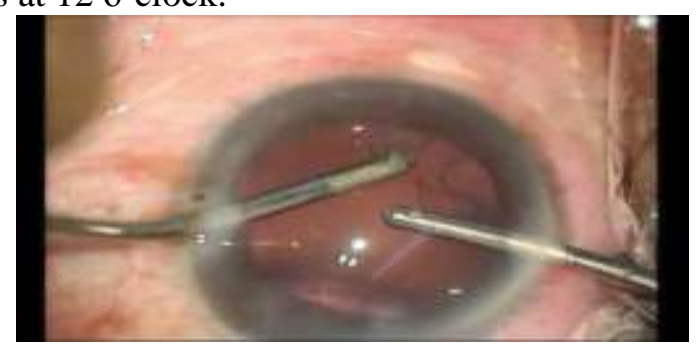

Figure (3): Irrigation and Aspiration.

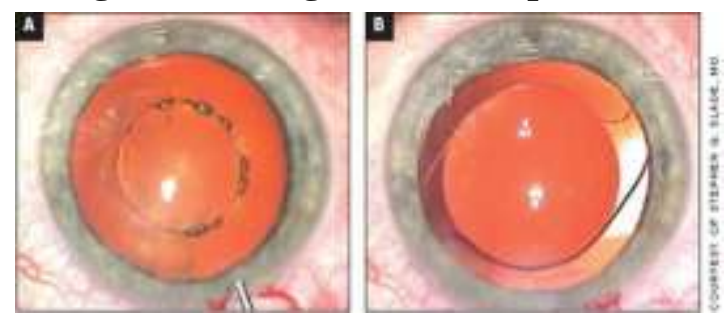

Figure (4): IOL implantation in bag.

\section{Trabeculectomy:}

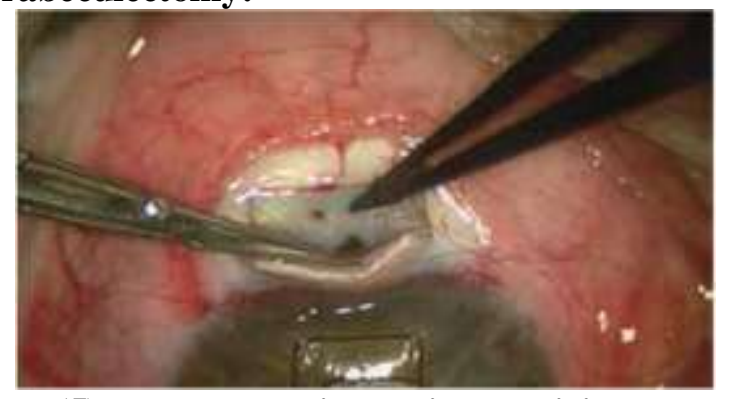

Figure (5): Trapdoor with peripheral iridectomy.

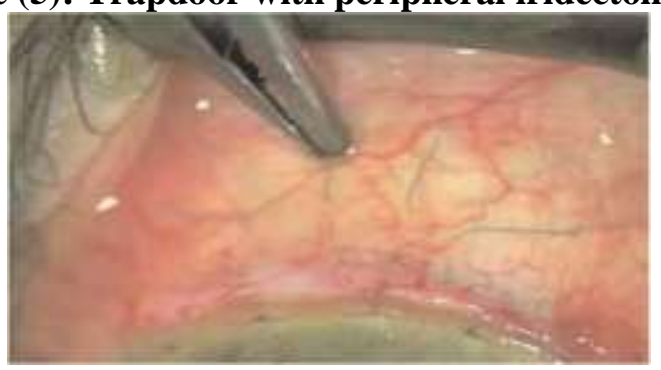

Figure (6): Closing of scleral and conjunctival flaps.
Preoperative measures were taken and the same previous anesthesia was done. Conjunctival flap (fornix based flap): Technique perio to my of $6 \mathrm{~mm}$ without releasing incisions advantages -easier to perform, disadvantages anterior leak is common, cautery applied to any bleeding episcleral vessels. Scleral Flap: Cut partial thickness flap typically 2-3 mm posteriorly by 4 $\mathrm{mm}$ wide. Raising the flap with crescent or pocket knife and extend it into clear cornea. Paracentes was performed in upper temporal peripheral cornea to allow the anterior chamber to be reformed. The Trapdoor (Figure 5), an incision 3-4 mm was made along the line of sclera spur into the anterior chamber and another incision was made $2 \mathrm{~mm}$ in front of it (rectangular window). This involve scleral spur, canal of schlemm, trabecular meshwork and peripheral cornea. The sclera window include small portion of the cornea to ensure that the whole trabecular meshwork and canal of schlemm were included in the excised portion. Iridectomy: To remove iris tissue from internal limbal opening to prevent blocking of internal opening by peripheral iris. The sclera flap was repositioned and sutured at 2 posterior corners (using 8/0 virgin silk), closing the conjunctiva, injection of BSS through paracentesis to test patency of fistula (Figure 6).

\section{Postoperative treatment:}

All patients received topical treatment in the form of antibiotic eye drops (moxifloxacin) five times daily, corticosteroid eye drops (prednisolone acetate) five times daily, and eye ointment (combination of antibiotic and steroid) once per day for one month. For group (A) patients, systemic corticosteroid and topical ciclopentolate were given to 2 cases which developed AC reaction. Four cases were reported for YAG laser posterior capsulotomy after 6 months. Anti glaucoma medication was given to one case. Anti haemorragic treatment (transxamicacid, alphachemo trypsin) and then vitrectomy was done. For group (B) patients, topical ciclopentolate was used 3 times daily and tight eye bandage and IV infusion of saline (Nacl 0.6) were given to the cases that developed hyoptony.

\section{Ethical considerations:}

The study protocol and all procedures performed involving human participants were approved by the Ethical Committee of the Faculty of Medicine, Al-Azhar University (Assiut), Egypt. Informed consent was obtained from all patients.

\section{Postoperative follow-up and evaluation:}

Patients were followed-up at the morning after surgery, as well as 1 week, 1 month, 3 months and 6 months postoperatively. A complete ocular examination 
was performed each time including visual acuity measurement, IOP measurement, slit-lamp examination, fundus and optic disc examination and optical coherence tomography on optic nerve head and ganglion cell layer, and contact A-scan biomicroscopy was performed at the $4^{\text {th }}$ visit to evaluate ACD and axial length changes. Also, complications were assessed.

\section{Statistical analysis:}

Data analyses were performed using IBM SPSS software V. 20.0. Data were tested for normality and for homogeneity prior to further statistical analysis. Categorical variables were described by number and percentage $(\mathrm{N}, \%)$, where continuous variables were described by the mean and standard deviation (Mean \pm $\mathrm{SD})$. Chi-square test was used to compare between groups regarding categorical variables where comparisons in continuous variables were done by paired t-test, independent sample t-test, repeated measures analysis of variance (ANOVA) and Bonferroni posttest. A two-tailed $p<0.05$ was considered statistically significant, however, $\mathrm{p}<0.01$ was considered as highly significant.

\section{RESULTS}

In this study, we evaluated fifty eyes of fortyeight patients (20 males and 28 females) with angle closure glaucoma with or without cataract, with an age range of 50:79 years. As we mentioned above they were divided into two groups: group (A) included 25 eyes underwent phacoemulsification with peripheral iridectomy, and group (B) included eyes underwent sub-scleral trabeculectomy.

No statistically significant differences were observed between groups in visual acuity at preoperative visit $(\mathrm{P}=0.107)$. Whereas, postoperative visual acuity of patients in phaco group was significantly increased (improved) compared to that of trabeculectomy group $(\mathrm{P}<0.001)$. Also, postoperative visual acuity of group (A) was significantly improved compared to preoperative one $(\mathrm{P}=0.049)$, this was evidenced by that $52.0 \%$ of cases had >0.4 postoperative VA. However, slight insignificant increase in postoperative VA was noticed in trabeculectomy group $(\mathrm{P}=0.896)$; Table 1 .

Table (1): Visual acuity between groups (pre and post-operative)

\begin{tabular}{|c|c|c|c|c|}
\hline \multirow[b]{2}{*}{ Visual acui } & & \multicolumn{2}{|c|}{ Groups } & \multirow[b]{2}{*}{$P$ value } \\
\hline & & $\begin{array}{c}\text { Group A (n=25) } \\
\text { N (\%) }\end{array}$ & $\begin{array}{c}\text { Group B }(n=25) \\
\text { N }(\%)\end{array}$ & \\
\hline \multirow{5}{*}{ Preoperative } & $<0.1$ & $3(12.0 \%)$ & $9(36.0 \%)$ & \multirow{5}{*}{0.107} \\
\hline & $0.1-0.2$ & $5(20.0 \%)$ & $7(28.0 \%)$ & \\
\hline & $0.2-0.3$ & $5(20.0 \%)$ & $5(20.0 \%)$ & \\
\hline & $0.3-0.4$ & $8(32.0 \%)$ & $2(8.0 \%)$ & \\
\hline & $>0.4$ & $4(16.0 \%)$ & $2(8.0 \%)$ & \\
\hline \multirow{6}{*}{ Postoperative } & $<0.1$ & $0(0.0 \%)$ & $7(28.0 \%)$ & \multirow{6}{*}{$0.001 * *$} \\
\hline & $0.1-0.2$ & $2(8.0 \%)$ & $6(24.0 \%)$ & \\
\hline & $0.2-0.3$ & $4(16.0 \%)$ & $6(24.0 \%)$ & \\
\hline & $0.3-0.4$ & $6(24.0 \%)$ & $4(16.0 \%)$ & \\
\hline & $>0.4$ & $13(52.0 \%)$ & $2(8.0 \%)$ & \\
\hline & \multicolumn{3}{|c|}{ Chi-square test was used. } & \\
\hline \multicolumn{5}{|c|}{ Table (2): Intraocular pressure between groups } \\
\hline \multirow{2}{*}{\multicolumn{2}{|c|}{ Intraocular pressure }} & Groups & & \multirow[b]{2}{*}{$P$ value } \\
\hline & & $\begin{array}{c}\text { Group A }(n=25) \\
\text { Mean } \pm \text { SD }\end{array}$ & $\begin{array}{c}\text { Group B }(n=25) \\
\text { Mean } \pm \text { SD }\end{array}$ & \\
\hline \multicolumn{2}{|c|}{ Preoperative } & $19.82 \pm 1.85$ & $20.11 \pm 2.89$ & 0.674 \\
\hline \multirow{4}{*}{ Postoperat } & 1 week & $13.23 \pm 1.26^{*}$ & $14.12 \pm 2.13^{*}$ & 0.078 \\
\hline & 1 Month & $13.15 \pm 1.28 *$ & $14.02 \pm 2.05^{*}$ & 0.079 \\
\hline & 3 Months & $13.28 \pm 1.36^{*}$ & $14.14 \pm 2.12 *$ & 0.094 \\
\hline & 6 Months & $13.69 \pm 1.35^{*}$ & $14.28 \pm 2.25^{*}$ & 0.266 \\
\hline
\end{tabular}

Independent $\mathrm{t}$-test and Paired t-test were used.

*: Statistically significant difference compared to pre-operative $(\mathrm{p}<0.05)$. 
There existed no obviously statistical significant difference between studied groups in IOP preoperatively and at all postoperative follow-up times (1 week, 1 month, 3 months and 6 months). While, at each time point after the surgery, IOP was improved in both group $\mathrm{A}$ and $\mathrm{B}$ as compared to preoperative value with statistical significance difference (all $\mathrm{P}<0.05$ ), IOP was decreased postoperatively by almost $30.0 \%$ in both groups A and B (Table 2).

The results showed no significant difference in preoperative anterior chamber depth (ACD) between groups $(\mathrm{p}=0.137)$, while, sub-scleral trabeculectomy group had significantly lower postoperative ACD (1.74 \pm 0.13) compared to phacoemulsification with peripheral iridectomy group $(3.14 \pm 0.23),(p<0.001)$. In group (A), there was a significant increase in postoperative ACD $(\mathrm{p}<0.001)$; Table 3 .
As regards the degree of angle closure after surgery (at 3 months), open angle was observed with no obvious goniosynaechiae presented in (group A), Goniosynachiae with angle closure $<180$ degrees appeared in (group B) and the degree of angle opening post operatively in all patients increased as compared with preoperative level. No significant difference was observed between groups in the preoperative angle opening distance (AOD) between the two groups $(\mathrm{p}=0.0714)$, whereas, there was a significant difference between groups in postoperative AOD $(\mathrm{p}<0.001)$. Postoperative AOD was significantly increased in both groups, the mean AOD in group (A) was $0.106 \pm 0.023$ preoperatively and increased postoperatively to $0.275 \pm$ 0.031 ( $\mathrm{P}<0.001)$, also, in group $(\mathrm{B})$, the mean preoperative AOD was $0.095 \pm 0.019$ and it significantly increased postoperatively to $0.115 \pm 0.028(\mathrm{P}<0.004)$; Table 3.

Table (3): Comparison between groups as regards ACD, AOD, Rim area, Rim volume, cup/disc area ratio and maximum cup depth

\begin{tabular}{|c|c|c|c|c|}
\hline \multirow{2}{*}{ Variable } & & \multicolumn{2}{|c|}{ Groups } & \multirow[b]{2}{*}{$P$ value } \\
\hline & & $\begin{array}{l}\text { Group A }(n=25) \\
\text { Mean } \pm \text { SD }\end{array}$ & $\begin{array}{c}\text { Group B }(n=25) \\
\text { Mean } \pm \text { SD }\end{array}$ & \\
\hline \multirow{3}{*}{$\begin{array}{l}\text { Anterior chamber } \\
\text { depth (ACD) }\end{array}$} & Preoperative & $1.81 \pm 0.15$ & $1.75 \pm 0.13$ & 0.213 \\
\hline & postoperative & $3.14 \pm 0.23$ & $1.74 \pm 0.13$ & $<0.001 * *$ \\
\hline & value & $<0.001 * *$ & 0.716 & \\
\hline \multirow{3}{*}{$\begin{array}{r}\text { Angle opening } \\
\text { distance (AOD) }\end{array}$} & Preoperative & $0.106 \pm 0.023$ & $0.095 \pm 0.019$ & 0.071 \\
\hline & postoperative & $0.275 \pm 0.031$ & $0.115 \pm 0.028$ & $<0.001 * *$ \\
\hline & value & $<0.001 * *$ & $0.004 * *$ & \\
\hline \multirow{3}{*}{$\operatorname{Rim} \operatorname{area}\left(\mathbf{m m}^{2}\right)$} & Preoperative & $1.15 \pm 0.59$ & $1.20 \pm 0.30$ & 0.707 \\
\hline & At 3 Months postop. & $1.15 \pm 0.56$ & $1.20 \pm 0.35$ & 0.706 \\
\hline & $\begin{array}{l}\text { At } 6 \text { Months postop. } \\
\text { alue }\end{array}$ & $\begin{array}{c}1.16 \pm 0.42 \\
0.912\end{array}$ & $\begin{array}{l}1.21 \pm 0.36 \\
0.915\end{array}$ & 0.653 \\
\hline \multirow{4}{*}{ Rim volume $\left(\mathrm{mm}^{3}\right)$} & Preoperative & $0.22 \pm 0.16$ & $0.23 \pm 0.10$ & 0.792 \\
\hline & At 3 Months postop. & $0.22 \pm 0.18$ & $0.23 \pm 0.11$ & 0.792 \\
\hline & At 6 Months postop. & $0.23 \pm 0.14$ & $0.24 \pm 0.12$ & 0.787 \\
\hline & value & 0.815 & 0.750 & \\
\hline \multirow{3}{*}{ Cup/disc area ratio } & Preoperative & $0.52 \pm 0.25$ & $0.50 \pm 0.25$ & 0.778 \\
\hline & At 3 Months postop. & $0.52 \pm 0.22$ & $0.50 \pm 0.22$ & 0.749 \\
\hline & $\begin{array}{l}\text { At } 6 \text { Months postop. } \\
\text { value }\end{array}$ & $\begin{array}{c}0.51 \pm 0.21 \\
0.879\end{array}$ & $\begin{array}{c}0.49 \pm 0.21 \\
0.822\end{array}$ & 0.738 \\
\hline \multirow{4}{*}{$\begin{array}{l}\text { Maximum cup depth } \\
(\mathrm{mm})\end{array}$} & Preoperative & $0.70 \pm 0.23$ & $0.68 \pm 0.21$ & 0.749 \\
\hline & At 3 Months postop. & $0.69 \pm 0.25$ & $0.67 \pm 0.19$ & 0.752 \\
\hline & At 6 Months postop. & $0.68 \pm 0.26$ & $0.66 \pm 0.20$ & 0.762 \\
\hline & alue & 0.919 & 0.940 & \\
\hline
\end{tabular}


Regarding Rim area, Rim volume, cup/disc area ratio and maximum cup depth, there were no statistically significant differences in comparison among preoperative, at 3 months, and at 6 months follow up "postoperative" of group (A) and also group (B), (almost the same values were noticed). Also, there were no significant differences in comparison between the two groups as regards preoperative, at 3 months and at 6 months follow up; Table 3.

Table (4): Postoperative complications between groups

\begin{tabular}{|c|c|c|c|c|}
\hline \multirow[b]{2}{*}{ Variable } & & \multicolumn{2}{|l|}{ Groups } & \multirow[b]{2}{*}{$P$ value } \\
\hline & & $\begin{array}{c}\text { Group A (n=25) } \\
\text { N (\%) }\end{array}$ & $\begin{array}{c}\text { Group B }(\mathbf{n}=25) \\
\text { N }(\%)\end{array}$ & \\
\hline \multirow{2}{*}{ Complications } & No & $17(68.0 \%)$ & $18(72.0 \%)$ & \multirow{2}{*}{0.757} \\
\hline & Yes & $8(32.0 \%)$ & $7(28.0 \%)$ & \\
\hline \multirow{5}{*}{$\begin{array}{l}\text { Type of } \\
\text { complication }\end{array}$} & Postoperative hypotony & $0(0.0 \%)$ & $6(85.7 \%)$ & \multirow{5}{*}{ 0.011* } \\
\hline & Vitreous hemorrhage & $1(12.5 \%)$ & $0(0.0 \%)$ & \\
\hline & Raised IOP & $1(12.5 \%)$ & $1(14.3 \%)$ & \\
\hline & Posterior capsule opacity & $4(50.0 \%)$ & $0(0.0 \%)$ & \\
\hline & Severe $\mathrm{AC}$ reaction & $2(25.0 \%)$ & $0(0.0 \%)$ & \\
\hline
\end{tabular}

Chi-square test was used.*: Significant $(\mathrm{p}<0.05)$

In Table (4), our study showed that there was no significant difference between thetwo groups regarding postoperative complications, 8 cases $(32.0 \%)$ in group (A) and 7 cases (28.0\%) in group (B) suffered from complications. As regard type of complication, postoperative hypotony contributed $85.7 \%$ of complications in group (B), whereas $50.0 \%$ of complications in group (A) was posterior capsule opacity (PCO), there was a statistically significant difference between studied groups regarding type of complications $(\mathrm{p}=0.011)$.

\section{DISCUSSION}

Primary angle-closure glaucoma is a main type of glaucoma, its multifaceted pathophysiology and various mechanisms contribute challenges for ophthalmologists. Angle-closure glaucoma is from the primary causes of blindness worldwide, it rising with age. The lens have an essential function in the pupillary block "the commonest pathogenic mechanism of PACG", consequently, removing the lens causes a high deepening of the chamber and reopening of the angle, this causes the elimination of the pupillary block and could prevent angle-closure glaucoma ${ }^{(3)}$. In the current study, we compared the clinical outcomes of phacoemulsification with peripheral iridectomy versus trabeculectomy in treatment of angle-closure glaucoma.

In the present study, as regards phacoemulsification in the management of angle closure glaucoma, postoperative visual acuity was significantly improved, ACD was increased and IOP was decreased by almost $33.0 \%$ at all times of postoperative follow-up.
Similar to our findings, Wang and $\mathbf{W} \mathbf{u}^{(2)}$ reported that after phacoemulsification, visual acuity was improved and IOP was decreased in patients with primary angleclosure glaucoma. Also, Bhandariet al. ${ }^{(11)}$ studied the effect of phacoemulsification on patients with glaucoma, they found that VA was improved, IOP decreased and anterior chamber depth increased postoperatively. Kurimoto et $\boldsymbol{a l} .^{(12)}$ reported a significant increase in ACD and trabecular-iris angle after phacoemulsification, especially in eyes with a narrower anterior chamber. In addition, Yang and Hung ${ }^{(13)}$ reported a significant increase in ACD and angle widening post extracapsular cataract extraction in patients with chronic PACG. These results present an evidence for lens participation in ACG pathogenesis and demonstrate positive effects of lens removal in PACG. Recently, it has been reported that primary lens extraction have an effective role in visual acuity improvement and long-term IOP control in PACG patients and this agreed with our study ${ }^{(\mathbf{1 4})}$.

Furthermore, Hayashi et al. $^{\left({ }^{(15)}\right.}$ found that anterior chamber depth and the width of the drainage angle in eyes with angle-closure glaucoma increased significantly after cataract extraction and intraocular lens implantation that could reduce IOP postoperatively. They added that the removal of a large cataractous lens from an eye with a crowded anterior segment may improve aqueous outflow. Also, it has been postulated that during phacoemulsification, the irrigating fluid flushes cellular debris from the trabecular meshwork, reducing the resistance to aqueous outflow and this agreed with our study ${ }^{(\mathbf{1 6})}$. The mechanism which causes 
IOP reduction after cataract extraction is not fully understood, but Huang et al. ${ }^{(3)}$ described a correlation between IOP reduction and angle widening after phacoemulsification. They reported that patients with narrow angle had lower IOP after phacoemulsification compared to those with open angles preoperatively. Huang et $\boldsymbol{a l} .{ }^{(\mathbf{1 7})}$ studied the association between preoperative lens vault and IOP reduction after cataract surgery. They concluded that preoperative lens vault could predict angle widening and intraocular pressure lowering after cataract surgery. In another study on 56 primary angle closure glaucoma patients who underwent phacoemulsification, Liu et al. ${ }^{(\mathbf{1 8})}$ demonstrated that preoperative measures of IOP and anterior chamber depth are positively correlated with long-term IOP control.

Regarding the results of trabeculectomy in the present study, we found that visual acuity and ACD almost not changed after the surgery, however, IOP was significantly decreased (it decreased by about $30 \%$ postoperative). Similar to our findings, Lochheadet $\boldsymbol{a l}{ }^{(8)}$ reported that trabeculectomy is effective in lowering intraocular pressure and could improve visual acuity in most PACG patients in short term but these patients may need IOP control by long-term. Chen $\boldsymbol{e t}$ al. ${ }^{(19)}$ found different surgical outcomes of trabeculectomy with adjunctive antimetabolites in eyes with acute primary angle closure glaucoma and chronic primary angle closure glaucoma, they found that surgical success (IOP $<21 \mathrm{HG}$ with or without medications) was lower in patients with APACG versus CPACG worse visual outcomes and postoperative complications were observed in APACG group. They concluded that trabeculectomy might not be the procedure of choice in medically unresponsive cases of APACG. In a study by Aung et al. $^{(\mathbf{( 2 )})}$, they reported a high risk of surgical failure (34.4\%) and complications with trabeculectomy without use of an adjunctive antimetabolite in medically unresponsive cases of acute primary angle closure, but the authors attributed high failure rates in these eyes to elevated preoperative IOP and associated inflammation. In addition, it has been reported that trabeculectomy has been shown to have an overall success rate of $68 \%$ in controlling IOP in PACG patients, any aqueous-draining procedure in an eye with a shallow anterior chamber and a chronic closed-angle poses the risk of further shallowing the anterior chamber or precipitating malignant glaucoma. It has been reported that trabeculectomy in PACG is associated with a higher risk of filtration failure, shallow anterior chamber, and malignant glaucoma/aqueous misdirection (9). Trabeculectomy increases the rate of cataract progression, and a significant proportion of patients will soon need cataract extraction after trabeculectomy ${ }^{(21)}$.

Our results showed a significant increase in VA after phacoemulsification as compared with trabeculectomy that showed little increase and significantly reduced IOP in both groups $(\mathrm{P}>0.05)$, after phacoemulsification and IOL implantation in patients with PAC there is statistically significant difference between pre and post-operative $(\mathrm{p}<0.001)$ in $\mathrm{ACD}$, but no statistically significant difference after trabeculectomy $(\mathrm{p}=0.716)$. Almost the same rate of postoperative complication was yielded (8 cases (32.0\%) in group A and 7 cases $(28.0 \%)$ in group B) suffered from postoperative complications, postoperative hypotony was the major complication in group (B) "85.7\%", whereas posterior capsule opacity (PCO) was noticed in $50.0 \%$ of group (A). Similar to our findings, Thamet al. ${ }^{(10)}$ compared phacoemulsification $(n=26$ eyes) versus trabeculectomy with adjunctive Mitomycin C(MMC) ( $\mathrm{n}=24$ eyes) in patients with medically uncontrolledchronic closed angle glaucoma(CACG) without cataract, both groups resulted in significant and comparable IOP reduction at 24 months postoperatively (34\% for phacoemulsifications and $36 \%$ for trabeculectomy) trabeculectomy group required on average 1.1 fewer drugs than phacoemulsification group $(\mathrm{p}<0.001)$ but had more postoperative complications ( $46 \%$ vs $4 \%$, $\mathrm{p}=0.001)$. The authors concluded that phacoemulsification alone may possible alternative to trabeculectomy as initial surgical intervention in medically uncontrolled CACG eyes without cataract. Also, Costa et al ${ }^{(\mathbf{9})}$ reported that trabeculectomy may cause complications related to hypotony and result in marked or total loss of visual acuity in patients with advanced field loss.

In a Cochrane review in 2006, Friedman and Vedula ${ }^{(22)}$ reported that both randomized trials and nonrandomized studies did not confirm the effectiveness of lens extraction for chronic primary angle-closure glaucoma. However, Lam et al. ${ }^{(\mathbf{2 3})}$ investigated cataract extraction in angle closure patients; they found that cataract extraction "with fewer medications" prevent the rise of IOP in acute angle closure patients. Tham $\boldsymbol{e t}$ $\boldsymbol{a l} .^{(24)}$ studied the effect of phacoemulsification as compared to phacoemulsification combined with trabeculectomy in chronic angle closure glaucoma patients, they found that while the IOP was slightly lower in patients with a combined procedure, patients who underwent phacoemulsification alone suffered from lesser complications and had no statistically significant differences in visual field progression in comparison with those underwent the combined procedure.

\section{Limitations of the study:}

This study has some limitations, of these; the sample size was relatively small and may have limited power of outcome comparisons. Moreover, there was no randomization in dividing patients and shortness of the follow-up period. 


\section{CONCLUSION}

The study found that both procedures are effective in lowering IOP in patients with angle-closure glaucoma, but phacoemulsification with peripheral iridectomy significantly improved visual acuity compared to sub-scleral trabeculectomy with almost the same rate of complications. Further studies with larger number of patients are required to confirm these findings.

Acknowledgements: We acknowledge all participants included in this study, besides the clinical staff members of Ophthalmology department, Al-Azhar University hospital,Assiut.

\section{REFERENCES}

1. World Health Organization(WHO)
impairment
apps.who.int/mediacentre/factsheets/fs282/en/index.html

2. Wang $\mathbf{F}, \mathbf{W u} Z$ (2016): Phacoemulsification versus combined phacotrabeculectomy in the treatment of primary angle-closure glaucoma with cataract: a Metaanalysis. Int J Ophthalmol., 9(4): 597-603.

3. Huang G, Gonzalez E, Peng PH, Lee R, Leeungurasatien T, He M, Porco T, Lin S (2011): Anterior chamber depth, iridocorneal angle width and intraocular pressure changes after phacoemulsification: narrow versus open iridocorneal angles, Arch Ophthalmol., 7 (2): 496-602.

4. Teekhasaenee C, Ritch $R$ (1999): Combined phacoemulsification and goniosynechialysis for uncontrolled chronic angle closure glaucomaafter acute angle closure glaucoma. Ophthalmol., 106:669-675.

5. Ang LP, Aung T, Chew PT (2000): Acute primary angle closure in an Asian population: long-term outcome of the fellow eye after prophylactic laser peripheral iridotomy. Ophthalmol., 107:2092-2096.

6. Cairns JE (1968):Trabeculectomy: Preliminary report of a new method. Am J Ophthalmol., 66:673-679.

7. Fernández S, Pardiñas N, Laliena JL, Pablo L, Díaz S, Pérez S, Honrubia FM (2009): Long-term tensional results after trabeculectomy: a comparative study among types of glaucoma and previous medical treatment. Arch SocEspOftalmol., 84:345-352.

8. Lochhead J, Casson RJ, Salmon JF (2003): Long-term effect on intraocular pressure of phacotrabeculectomy compared to trabeculectomy. Br J Ophthalmol., 87, 850852.

9. Costa VP, Smith M, Spaeth GL, Gandham S, Markovitz B (1993): Loss of visual acuity after trabeculectomy. Ophthalmol., 100:599-612.

10. Tham CY, Kwong YY, Baig $\mathbf{N}$ et al. (2013): phacoemulsification versus trabeculectomyin medically uncontrolled chronic angle closure glaucoma without cataract. Ophthalmol., 120:62-67.
11. Bhandari S, Pandyal I, Khanal SP, Thapa SS (2014): Effect of phacoemulsification surgery on various parameters in patients with glaucoma. Nepal $\mathbf{J}$ Ophthalmol., 6 (11):46-55.

12. Kurimoto Y, Park M, Sakaue H, Kondo T (1997): Changes in the anterior chamber configuration after smallincision cataract surgery with posterior chamber intraocular lens implantation. Am J Ophthalmol., 124:775-780.

13. Yang CH, Hung PT (1997): Intraocular lens position and anterior chamber angle changes after cataract extraction in eyes with primary angle-closure glaucoma. J Cataract Refract Surg., 23:1109-1113.

14. Moghimi S, Hashemian $H$, Chen $R$, MohammadkarimJohari, MassoodMohammadi, Shan C, Lin B (2015): Early phacoemulsification in patients with acute primary angle closure. J Current Ophthalmol., 27: 70-75.

15. Hayashi K, Hayashi H, Nakao F, Hayashi F (2001): Effect of cataract surgery on intraocular pressure control in glaucoma patients. J Cataract Refract Surg., 27(11):1779-86.

16. Baillif S, Roure-Sobas C, Le-Duff F, Kodjikian L (2012): Aqueous humor contamination during phacoemulsification in a university teaching hospital. J FrOphtalmol., 35(3):153-156.

17. Huang G, Gonzalez E, Lee R et al (2012): Association of biometric factors with anterior chamber angle widening and intraocular pressure reduction after uneventful phacoemulsification for cataract. J Cataract Refract Surg., 38(1):108-116.

18. Liu CJ, Cheng CY, Ko YC et al. (2011): Determinants of long-term intraocular pressure after phacoemulsification in primary angle-closure glaucoma. $\mathbf{J}$ Glaucoma,20(9):566-570.

19. Chen YH, Lu DW, Cheng JH et al. (2009):Trabeculectomy in patients with primary angle closure glaucoma, 18:679-683.

20. Aung T, Tow SL, Yap EY et al. (2000):Trabeculectomy for acute primary angle closure. Ophthalmol., 107:12981302.

21. Andrew S, Tsai H, Pui YB, Htoon HM, Wong TT (2015): Bleb needling outcomes for failed trabeculectomy blebs in Asian eyes: a 2-year follow up. Int J Ophthalmol., 8(4): 748-753.

22. Friedman DS, Vedula SS (2006): Lens extraction for chronic angle-closure glaucoma. https://www.ncbi.nlm.nih.gov/pubmed/16856103

23. Lam DS, Leung DY, Tham CC et al. (2010): Randomized trial of early phacoemulsification versus peripheral iridotomy to prevent intraocular pressure rise after acute primary angle closure. Ophthalmol., 115(7):1134-1140.

24. Tham CC, Kwong YY, Leung DY et al.(2008): Phacoemulsification versus combined phacotrabeculectomy in medically controlled chronic angle closure glaucoma with cataract. Ophthalmol., 115(12):2167-2173. 